$$
\begin{aligned}
& \text { توزيع ترسبات الترب في تركيب بعثيقة شمال مدينة الموصل } \\
& \text { باستخدام معطيات التحسس النائي }
\end{aligned}
$$

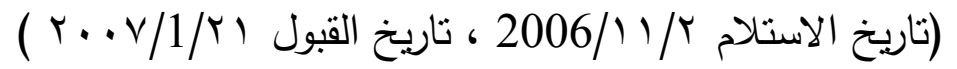

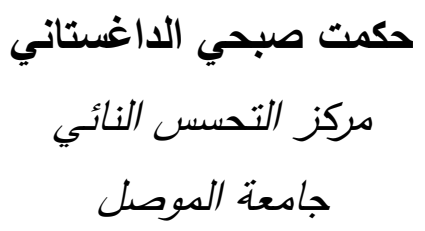

الملخص

استخدمت معطيات التحسس النائي في دراسة توزيع ترسبات الترب المنتكلة بترسبات العصر الرباعي في تركيب بعشيقة شمال مدينة الموصل. اسفرت الدراسة عن تمييز ثلاثة اصناف رئيسة بالاعتماد على لئ لئي منشئها التكويني، الى جانب ذلك تتفل عن الصنفين الاولين خمسة اصناف ضمنية اعتمادا على موقعها والتي يثكل فيها العامل الجيومورفولوجي اساس في التزيع المكاني لترسبات التزب على الخارطة الغرضية التي أعدت بمقياس 1/50,000.

تم تعزيز هذه الخارطة عن طريق تحليل المرئية الفضائية نوع (TM) بمقياس 1/100,000 بالاعتماد على البرامجيات المتوفرة في التصنيف. نم فصل هذه الاصناف عن طريق مقارنة الانعكاسية الطيفية للخلايا الصورية للمرئية مع البصمة الطيفية لكل خلية صورية مختارة للصنف الواحد.

اظهرت مكاملة ومناقتشة النتائج وتوثيق التصنيف اعلاه بالعمل الحقلي والمختبري، لغرض تثثيت الفروقات بين هذه الاصناف، الى جانب توضيح اسباب اختلاف الانعكاسية الطيفية من موقع الى آخر.

\title{
Distribution of Soil Deposites in Bashiqa Structure, North of Mosul City, Using Remote Sensing Data.
}

\author{
Hekmat S. Al-Daghastani \\ Remote Sensing Center \\ Mosul University
}

\section{Mayada M. Al-Dulaimi}

Department of Earth Sciences

College of Science/Mosul Universit

\section{ABSTRACT}

Remote sensing data were employed to study the soil distribution of Late Quaternary deposits in Bashiqa Structure, north of Mosul City. The outcome of this study distinguished three primary classes of soils depending on their morphogenetic origin. 
Furthermore, the first two classes encompass five subclasses related to its particular site within the study area, and in which the geomorphological factor plays an important role in the spatial distribution of soil forms; thus providings the tools of soil classification distribution map at the scale of $1 / 50,000$.

The map produced is commensurable with the analysis of Landsat imagery at $1 / 100,000$ scale, which resulted in the development of thematic image classified according to the relevant available computer programs. The separation of these classes accomplished through comparison of reflected spectrum of the optical pixels of the images with the spectral signature of each image pixels related to individual class.

The integration and discussion of the results and documentation of the above mentioned classification were ascertained by field and laboratory examinations in order to verify the differences among these classes and to enhance the variability of spectral reflectance from one site to another.

\section{المقدمة}

نظرا لاهمية التربة في ديمومة الحياة على سطح الكرة الارضية، فقد نشأ العديد من الاختصاصات في علم التربة ومن اهمها علم البيديولوجيا (Pedology) الذي يهتم بدراسة التربة من حيث منشئها، تطورها، وخواصها ومن ثم تصنيفها (Soil Survey Staff, 1975). استطاع (Buringh, 1960) من تمييز ثناثة مجاميع ترب عظمى(Great Soil Groups)، فكانت باكورة دراسته بوضع خريطة اولية للعراق توضح تصنيف الترب وبمقياس 1/1000,000. اشار هذا الباحث الى محتواها الكيمياوي، الحياتي، وطبيعتها الفيزياوية، وكذلك الى العوامل المؤثزة في طبيعة الاختلافات بين هذه الانواع الرئيسة والتي من اجلها صنفت الترب في العراق الى (1 ( ) صنفاً ـ يوضح الثكل (1) جزء من خارطة العراق لتصنيف الترب لنتمل منطقة الدراسة. لاتوجد دراسات حقلية أو مختبرية تفصيلية دقيقة حول انواع الترب في تركيب بعشيقة والمناطق التي تحيط بها عدا الدراسة التي قام بها (العبادي، ؟ . . ب)، اذ اخذ الباحث نماذج من مواقع عدة ومن ضمنها نماذج من منطقة المقدمات الجبلية والتي تشمل تراكيب عين الصفرة، مقلوب، بعثيقة حيث اكد فيها على الخواص الطبيعية الحقلية للاستخدامات الرعوية.

تشمل الترسبات السطحية للعصر الرباعي(Quaternary deposits) والمحيطة بتركيب بعشيقة بشكل رئيس من مواد مفككة ضعيفة التماسك ذات زوايا حادة الى متوسطة الاستدارة، ومتمتلة بسطوح المراوح الفيضية (Alluvial fans) التي تحتوب على خليط من المواد غير المصنفة مضافا اليها المواد المنهالة (الهابطة) (Mass wasting) التفاضلية ونشاط وحركة الصدوع والفواصل في تركيب بعشيقة. الى جانب ذلك تتواجد نرسبات السهول الفيضية وترسبات مليء الوديان (Flood plain and valley filling)، التي تشكل احزمة مختلفة المساحات 
تحيط بالمناطق الواقعة ضمن وجوار قنوات الوديان الموسمية المنحدرة من تركيب بعشيقة -Salih and Al)

.Daghastani, 1993)

تهدف الدراسة الحالية الى ادخال تقنية التحسس النائي والمتمنكة بالتفسير البصري والرقمي للمعطيات

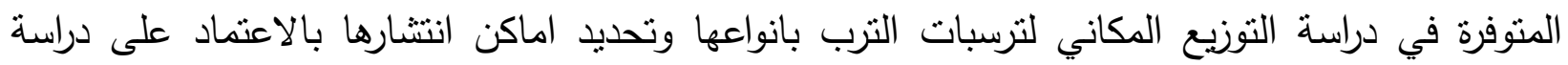
وتحليل مظاهر اثكال سطح الارض وطرائق استخداماتها الى جانب دراسة طبيعة تباين الانعكاسية الطيفية

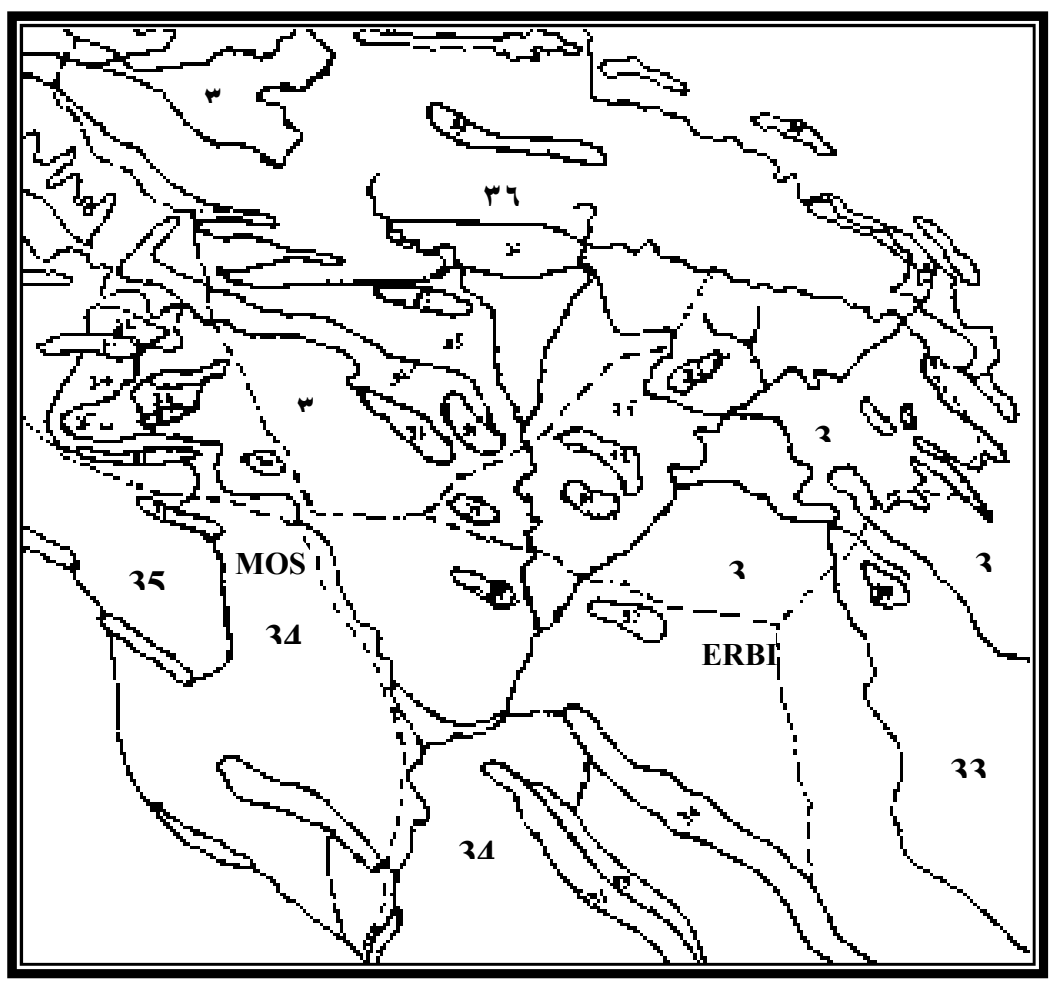

لكل صنف من هذه الاصناف.

الثكل ا : جزء من خارطة العراق لتصنيف الترب لتتشمل منطقة الدراسة، عن(Buringh, 1960).

اولا: التصنيف بالاعتماد على الصور الجوية البانكروماتية: يتضح من تحليل الدراسات السابقة والمنجزة في مجال تصنيف الترب تحت ظروف مختلفة ان سطح التربة عبارة عن جسم طييعي ديناميكي متطور على سطح الارض ولها ثلاثة ابعاد (مساحة وعمق) تكونت

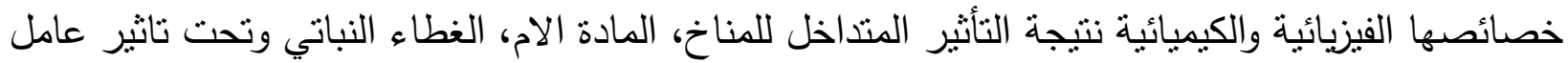
الانحدار لازمنة متباينة (الاغستاني، ؟ . .ץ). ان عملية التغيير في عنصر واحد من العوامل اعلاه ممكن ان ينشئ انواعا معقدة من الترب، وبذللك يجب اخذها بالحسبان عند القيام بتصنيف الترب، اذ انها تعتبر العمود الفقري تبعا لتاثرها بالعمليات الجيومورفولوجية الظاهرية السائدة في منطقة الدراسة (Bruce, 1975).

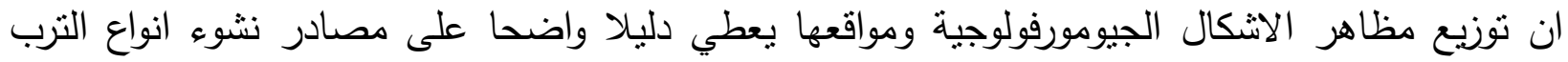
التي تغطيها (Mulders, 1987). 
نظرا للترابط الوثيق بين مظاهر الاشكال الجيومورفولوجية واستخدامات الارض و الغطاء الارضي من

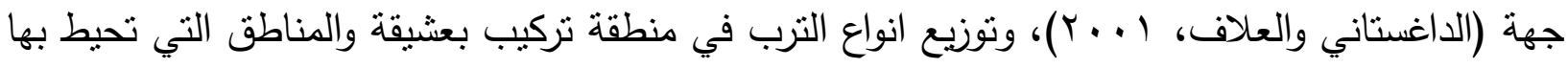

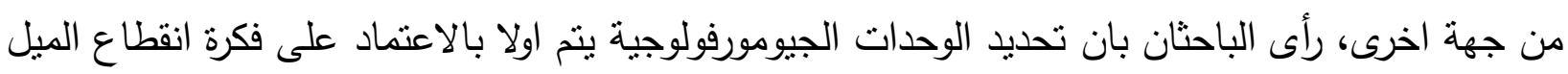

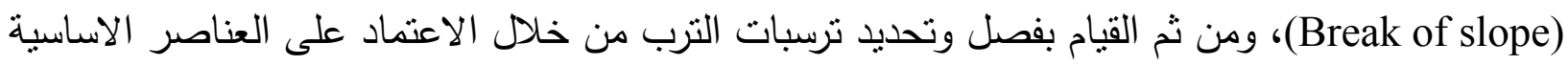

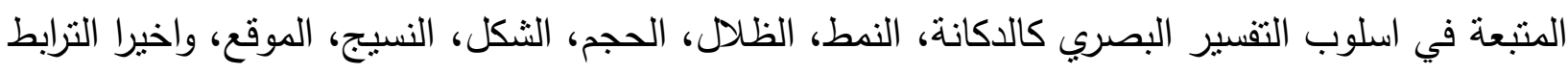

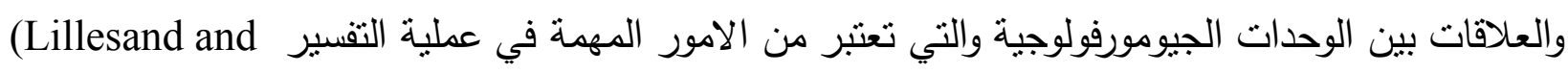
.Kiefer, 2000)

يوضح الثكل (r) خارطة تصنيف ترسبات الترب النهائية في منطقة بعشيقة والمفسرة من الصور

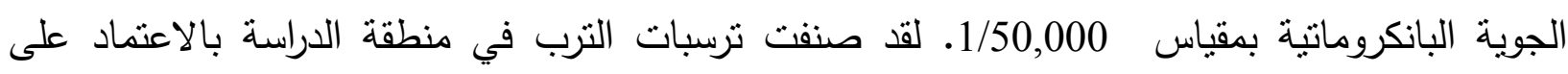

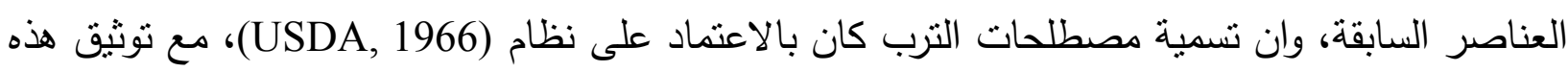
الاصناف (Classes) بالنماذج المختبرية لكل صنف والتي سوف نتطرق اليها في الفقرات القادمة، اذ امكن تمييز ثلاثة اصناف رئيسة:

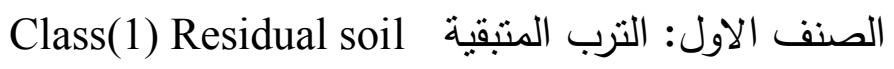

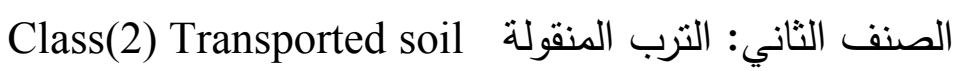

الصنف الثالث: الترب التجميعية المتعددة المنشأ Class(3) Polygenetic soil قسم الصنف الاول والثاني الى اصناف ضمنية (Sub-classes) وذلك لظهور اختلافات بسيطة ضمن

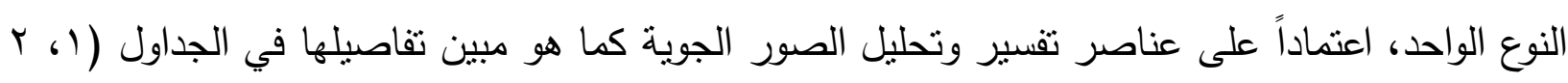

الجدول ا : الصنف الاول، الترب المتنقية (Residual Soil) , (1)

\begin{tabular}{|c|c|c|c|c|c|c|}
\hline الدكانة & النسيج & الموقع & الثكل & نوع المواد & العمليات & \\
\hline داكن & ناعم & الجزء الأعلى من تركيب & 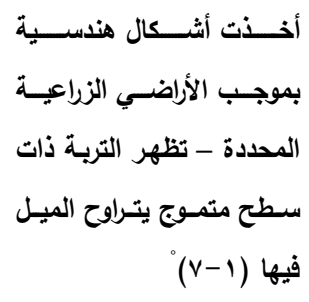 & 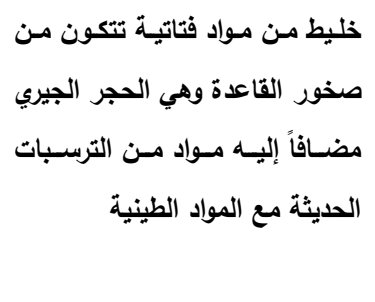 & كيمياوية & $\begin{array}{c}\text { Subclass } \\
\text { (1) }\end{array}$ \\
\hline متوسط & متاعم الى & 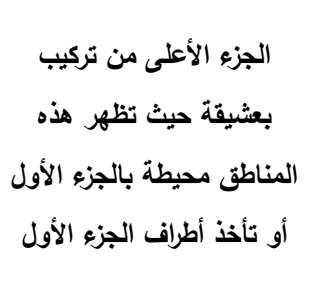 & 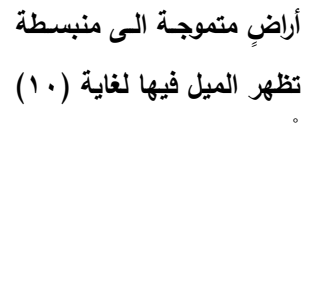 & 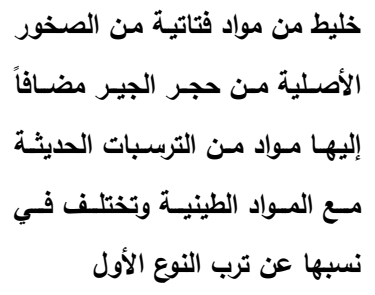 & كيمياوية & $\begin{array}{l}\text { Subclass } \\
\text { (2) }\end{array}$ \\
\hline
\end{tabular}


توزيع ترسبات الترب في تركيب بعشيقة شمال مدينة الموصل.............

الثكل r : خارطة تصنيف ترسبات الترب في تركيب بعشيقة والمفسره من الصور الجوية.

الجدول r : الصنف الثاني، الترب المنقولة Class (2), Transported Soil 
حكمت صبحي الاغستاني و ميادة محمود الدليمي

\begin{tabular}{|c|c|c|c|c|c|c|}
\hline الدانة & النسيج & الموقع ل & الثكل - الثل & نوع المواد & العمليات & التسمية \\
\hline تلترج الترب من الفاتحة & (حسب تدرج المواد & 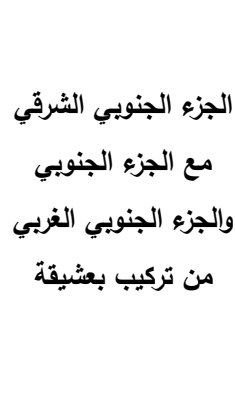 & وذات أثكال مخروطية & 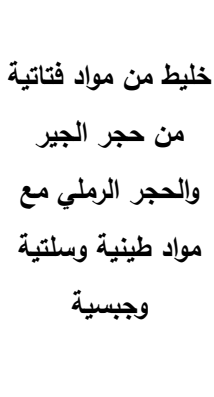 & 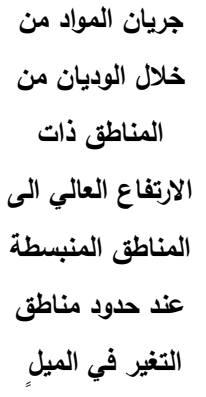 & $\begin{array}{c}\text { ترب المراوح } \\
\text { (Subclass) } \\
\text { (1) }\end{array}$ \\
\hline يتكرج من متوسط الى & يتلرج من متوسط & 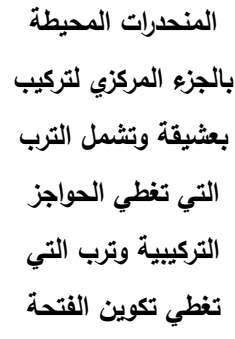 & أثنكال المنددرات & 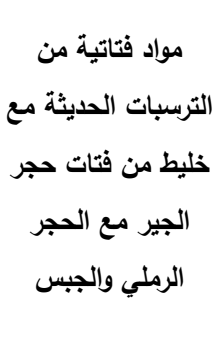 & 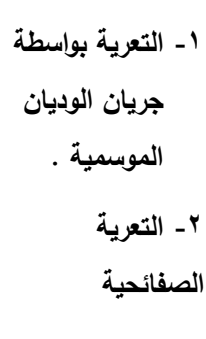 & $\begin{array}{c}\text { ترب } \\
\text { (Subclass) } \\
\text { (2) }\end{array}$ \\
\hline من فاتح الى متوسط & ناعم & تعمل شكل شريط & 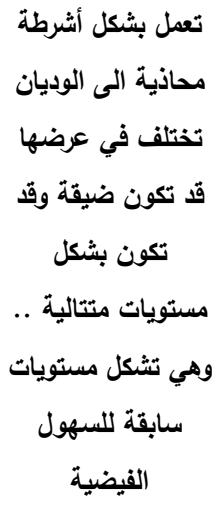 & 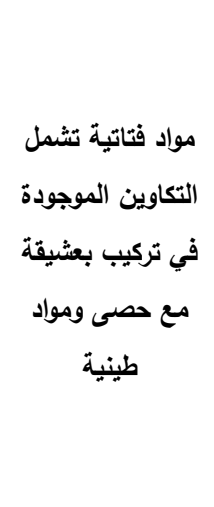 & 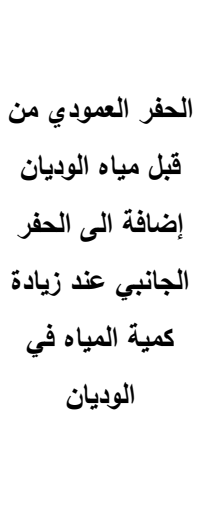 & $\begin{array}{c}\text { ترب المدرجات } \\
\text { (Subclass) } \\
\text { (3) }\end{array}$ \\
\hline
\end{tabular}

Class (3) Polygenitic Soil الجدول ب : الصنف الثالث، الترب التجميعية المتعددة المنثأ

\begin{tabular}{|c|c|c|c|c|c|c|}
\hline الدكانة & النسيج & الموقع & الثكل & نوع المواد & العطليات & الاسم \\
\hline 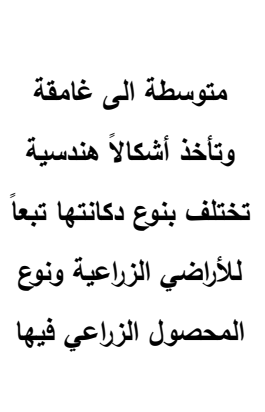 & ناعم الى & 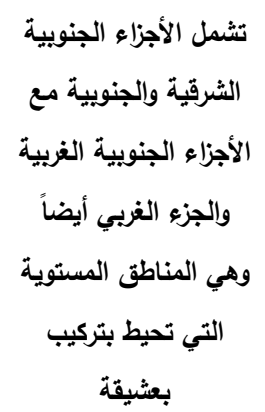 & $\begin{array}{c}\text { تراب منبسطة فيها العيل } \\
\text { يتراوح }(v-1)\end{array}$ & 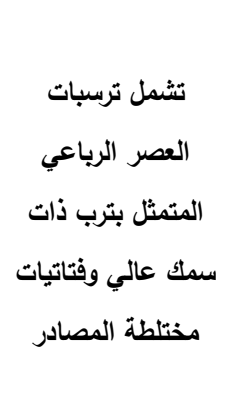 & . & $\begin{array}{c}\text { التجربية } \\
\text { class (3) } \\
\text { التجميعي }\end{array}$ \\
\hline
\end{tabular}

ثانيا : التصنيف بالاعتماد على المرئيات الفضائية: 
ان عملية مسح وتصنيف ترسبات الترب بالاعتماد على المرئيات الفضائية هي محاولة لجعل الخلايا

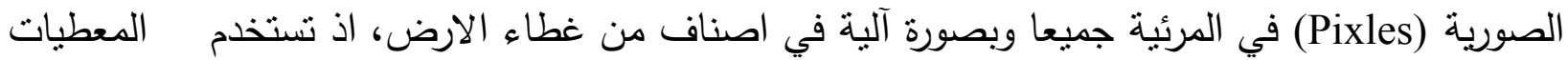
المتعددة الاطياف عادة لاتجاز هذا العمل. ان المدى الاعتيادي لقيم المقادير الطيفية المنعكسة من

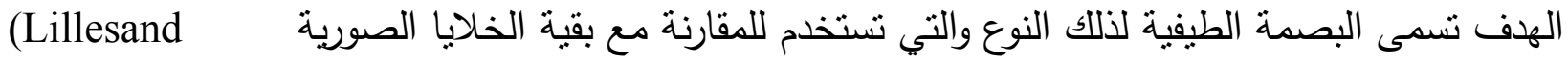
.and Kiefer, 2000)

تم تصنيف جزء من مرئية فضائية لتركيب بعثيقة والمناطق المستوية المحيطة بها والملتقطة بواسطة راسم الخرائط الموضوعي للقمر الاصطناعي الامريكي لاندسات لثنلاث قنوات طيفية لتقطة بتاريخ

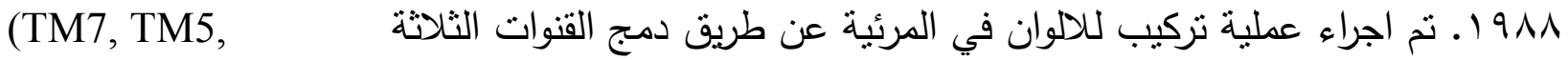
(AL-Shumam, 2001) لاغراض التصنيف. نم اختيار (ISMIC) (المعد من قبتخدام برنامج (TM3) نماذج من المرئية ممثلة بمناطق منتخبة ضمن مناطق ترسبات الترب الموجودة بموجب تعدد الانعكاسات

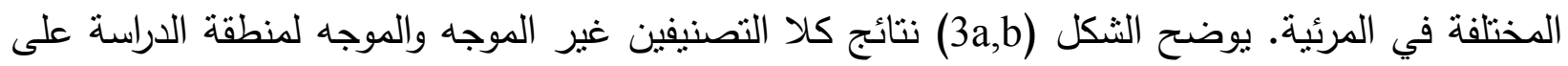

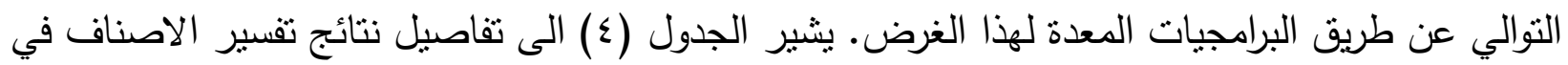
المرئية الفضائية.

لوحظ من خلال تحليل المرئية المعاملة بالتصنيف الموجه وغير الموجه وجود تكرار لوني لعدد من

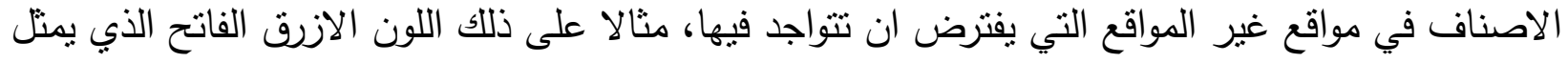

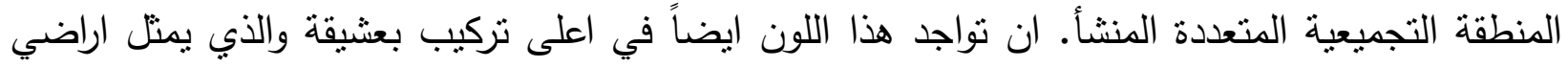
غير مزروعة مختلطة بالمواد الفتاتية من الصخور الاصلية (Subclass 2) من ترسبات الترب المتنقية، وهذا

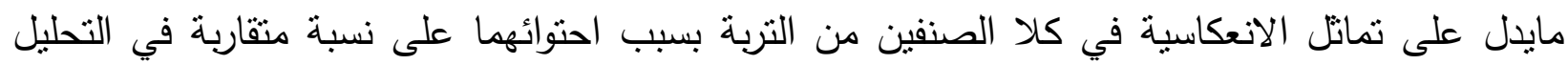
المختبري من حيث نسبة الكلس، المادة العضوية، الجبسوم وكذلك في صنف النسيج (Texture class) لهذه التربة والتي سوف نتطرق اليها في الفقرة القادمة.

الجدول ع : نتائج تفسير أصناف الترب في المرئية الفضائية.

\begin{tabular}{|c|c|c|}
\hline الوصف & اللون & الصنف \\
\hline ترب متبقية في أعلى تركيب بعثيقة ، تمثل ترب مزروعة بالمحاصيل & 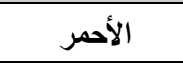 & $(1$ \\
\hline حواجز تركيبية (المكاشف الصخرية لحجر الكلس) . & الأخضر & $(r$ \\
\hline 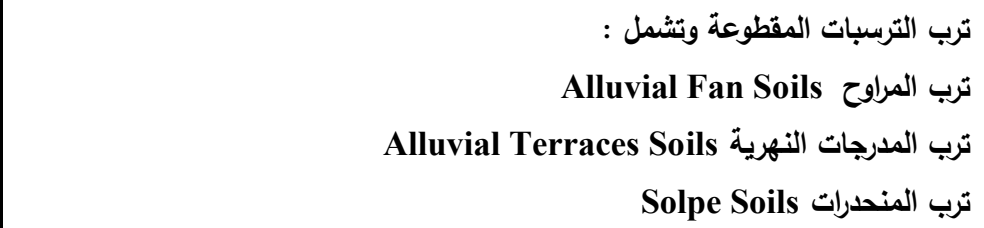 & الأزرق الغامق & $(\mu$ \\
\hline ترب متبقية في أعلى تركيب بعثيقة وهي تمثل ترب مزروعة. & الأصفر & $(\xi$ \\
\hline التزب التجميعية العميقة والتي تكون متعدة الثصادر والمحيطة بتركيب بعثيقة . & الأزرق فاتح & $\left(0^{\circ}\right.$ \\
\hline تروب سميكة ذات نسبة عالية من العواد العضوية وتستخذم لزراعة الأثجار وتتواجد في & الوردي & $(9$ \\
\hline
\end{tabular}


حكمت صبحي الداغستاني و مبادة محمود الدليمي

الثكل (3a,b) : تصنيف ترسبات الترب بالاعتماد على المرئيات الفضائية.

ثالثا : النماذج الحقلية وتحضيرها للتحاليل المختبرية: 
من خلال زيارة منطقة الدراسة ميدانيا والتعرف على ظروف المنطقة من الناحية الجيومورفولوجية

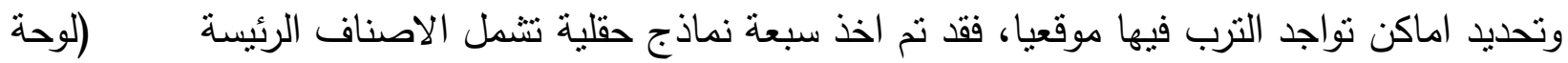

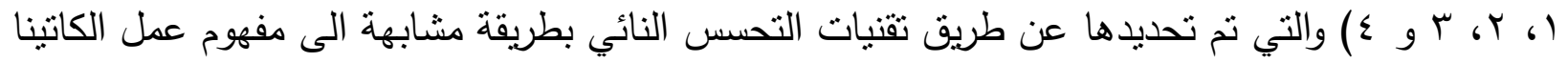
والذي يمكن ان يعوض عن طريقة عمل مقاطع الترب (Catena) (Soil profile) في المنطقة (Biswas) and Gawande, 1964)

$$
\text { الزراعة والغابات (الدليمي، } 0 \text {. . ب). }
$$

تتضمن نتائج تحليل الصفات الفيزياوية والكيمياوية لنماذج الترب من المواقع المدروسة (الجدول ه) بعض الصفات المشتركة بين النماذج وهي درجة الحموضة، التوصيل الكهربائي، اذ يمكن ملاحظة التقارب في درجات التفاعل فهي متعادلة الى قليلة القاعدية نسبيا. اما فيما يخص التوصيل الكهربائي فان انخفاض القيم يدل على ان الترب غير ملحية، بلغت نسب الجبسوم القيم الوسطية تقريبا في جميع النماذج اذ نتلغ اعلى نسبة للجبسوم هي (2.064) والمتأثزة بمادة الام (مكاثف نرسبات تكوين الفتحة) في نموذج المنحدرات والتي تقع ضمن المواصفات المشار اليها في تحاليل مشروع ري الجزيرة الشرقي الذي يغطي جزء من منطقة الدراسة (Nedeco, 1982).

الجدول 0 : نتائج التحليلات المختبرية لنماذج الترب في منطقة بعشيقة.

\begin{tabular}{|c|c|c|c|c|c|c|c|c|c|c|c|}
\hline \multirow{2}{*}{ النسجة } & \multicolumn{3}{|c|}{ \% مفصولات التربة } & \multirow{2}{*}{ \% المسامية } & \multirow{2}{*}{ الظثافة } & \multirow{2}{*}{ \% الجبس } & \multirow{2}{*}{ \% العضوية } & \multirow{2}{*}{$\operatorname{caco}_{3} \%$} & \multirow{2}{*}{$\begin{array}{c}\text { التوصيل } \\
\text { Ec } \\
\text { ds.m }{ }^{-1}\end{array}$} & \multirow{2}{*}{$\begin{array}{c}\text { درجة } \\
\text { التفاعل } \\
\text { PH }\end{array}$} & \multirow{2}{*}{ مواقع العينات } \\
\hline & طين & سلت & رمل رمل & & & & & & & & \\
\hline طينية & Tr.Y & ro & IY.A & $\varepsilon r$ & 1.0 & 1.rVY & .997 & $r \leq .11$ &.$r \Delta q$ & v. & بعثيقة (1) \\
\hline طينية & $\varepsilon V . r$ & $r V$ & ro.1 & $\varepsilon$. & 1.7 & $1 . . r r$ & .r. $\theta$ & rv.vo &..$\leqslant r_{0}$ & V.o & بعشيقة (r) \\
\hline مزيجية & YV.r & $r v$ & ro. & $\varepsilon V$ & $1 . \varepsilon$ & $r . .+Y \leq$ &. $.7 \wedge \wedge$ & $r \leqslant .04$ & .091 & v. 4 & نموذج المنحدر \\
\hline مزيجية & rV.r & rr & $\leq . . \wedge$ & $\leq v$ & $1 . \varepsilon$ & I.Vr. & 1.97. & rr.V $\leq$ & .rq. & V.o & المراوح الفيضية \\
\hline مزيجية & rV.r & $r_{1}$ & $\leqslant 1.1$ & $\varepsilon$. & 1.7 & I.VY. &..$I V Y$ & rv.rq & .roq & V. & المدرجات النهرية \\
\hline مزيجية & rV.r & YI & $\leqslant 1.1$ & $\varepsilon$. & 1.7 & I.VY. & 1.717 & rr.r & . tro & V.乏 & المنطقة التجميعية \\
\hline طينية & $\varepsilon V . r$ & \& & $1 . .1$ & $\varepsilon$. & 1.7 & I.Vr. & $. r \leq r$ & rv.vo & מדזיט & V.. & المنطقة التجميعية \\
\hline
\end{tabular}


يوضح الثكل (ع) العلاقة المنبادلة بين نتائج التحليلات الفيزياوية ومواقع نماذج التربة، اذ يتبين ان

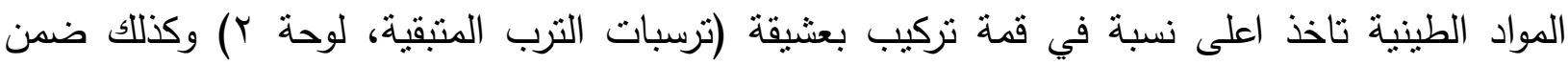
ترسبات ترب المناطق التجميعية (لوحة () بسبب قلة الانحدار، ويتذبذب المنحي بين مواقع المنحدرات

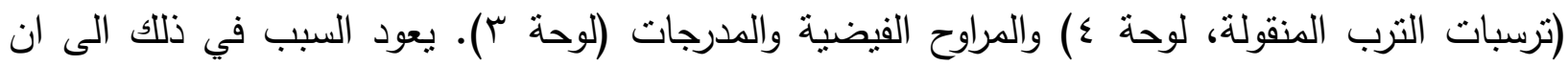
المواد الطينية المنواجدة في نماذج اشكال المنحدرات (ترسبات ترب المنحدرات، المراوح والمدرجات)، تغسل

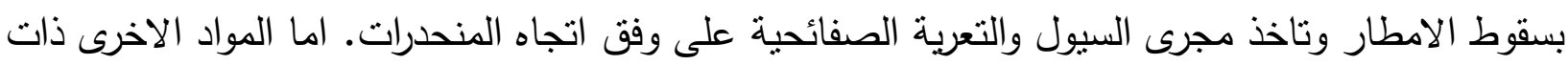
الدقائق الاكثر خشونة فانها تتذبذب، ولكن تبلغ اقصى قيمة لها في مواقع الترسبات النهرية مثل المدرجات

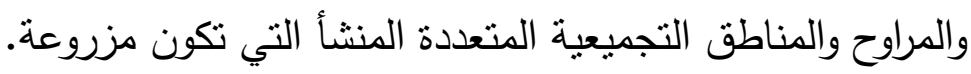

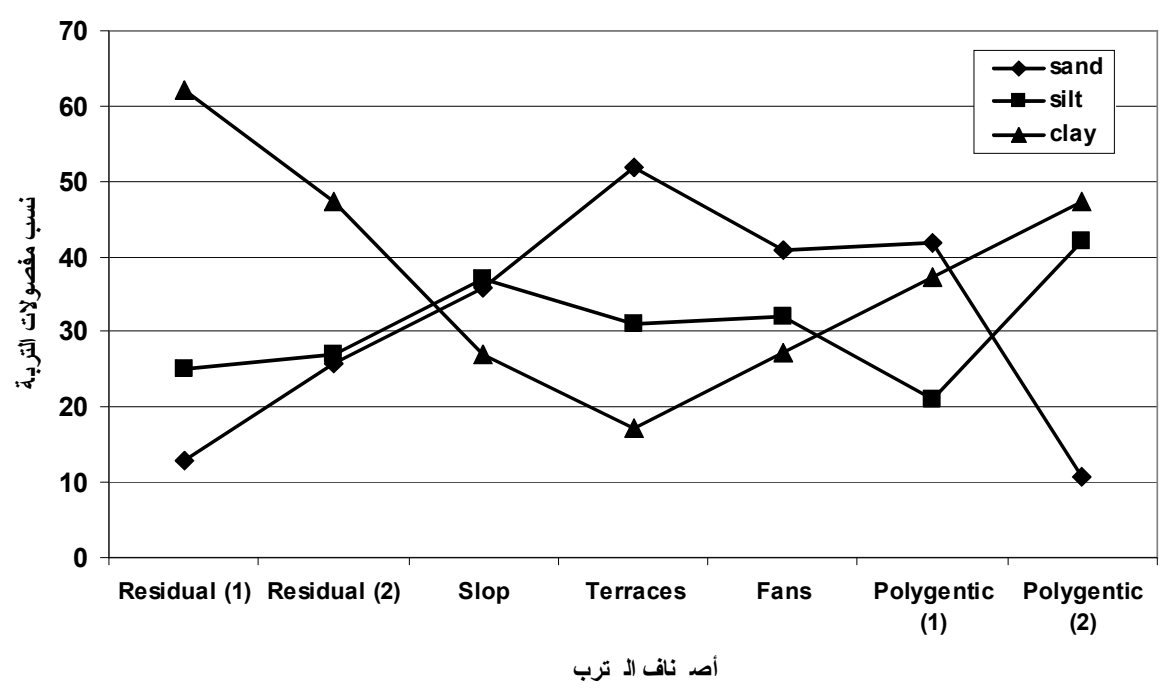

الثكل ؟ : منحنيات مفصولات التربة وعلاقتها مع مواقع ترسبات اصناف الترب. 
توزيع ترسبات الترب في نركيب بعشيقة شمال مدينة الموصل.............

لوحة ؟ : الترب المنبقية في اعلى تركيب بعشيقة والمستخدمة في زراعة الحبوب...

لوحة س: الترب المنقولة (ترب المدرجات والمراوح الفيضية ) قرب مدينة بعشيقة والمستخدمة في زراعة اشجار الزبتون. 
اما فيما يخص المواد العضوية، فيوضح الثكل (0) هذه العلاقة، اذ تبلغ اعلى نسبة لها في المراوح

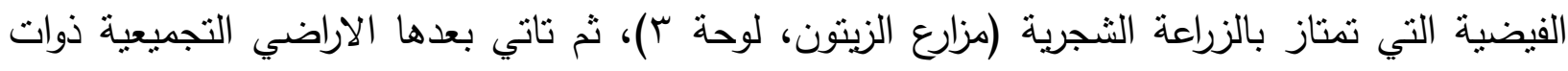

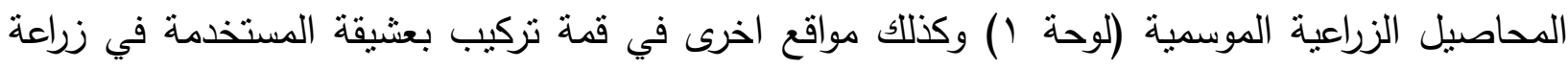
محصولي الحنطة والثعير (لوحة ب).

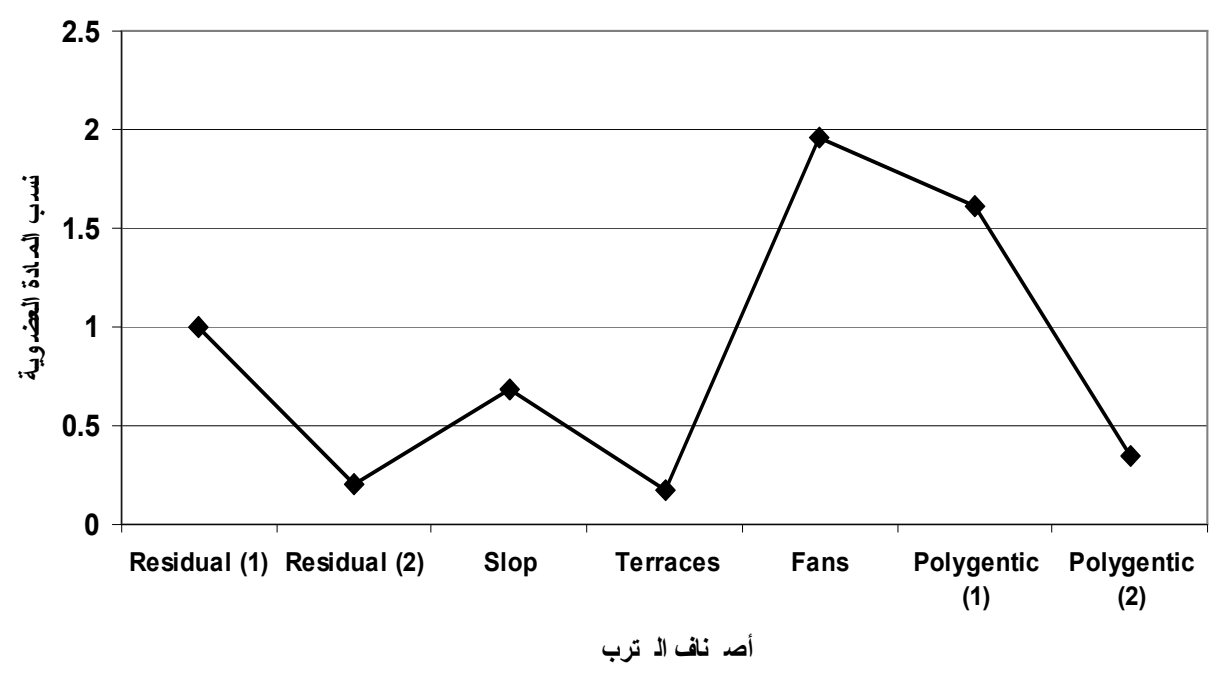

الثكل ه : منحني المادة العضوية وعلاقته مع مواقع ترسبات اصناف الترب.

لوحة ؟ : الترب المنقولة (ترب المنحدرات والمراوح الفيضية ) قرب مدينة بحزاني. 


\section{الاستنتاج}

'. تم استحداث خارطة لتوزيع وتصنيف ترسبات الترب بالاعتماد على معطيات التحسس النائي لمنطقة

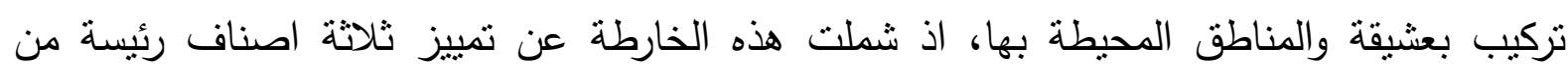
ترسبات الترب الى جانب خمسة اصناف ضمنية اخرى. اثتت الفحوصات المختبرية والكيميائية تبعية كل صنف من اصناف الترب الى موقعه الجيومورفولوجي، وذللك عن طريق التحري عن اسباب تاثير التئ الفحوصات المختبرية في تباين الانعكاسية الطيفية بين الاصناف اعلاف التهاه

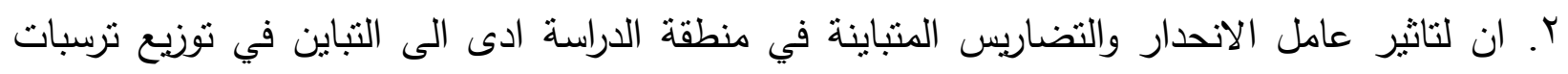

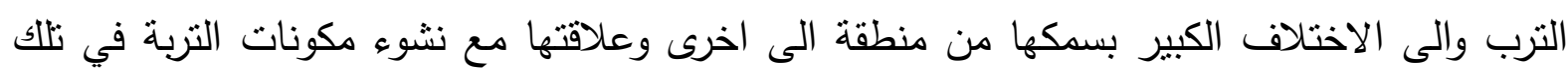

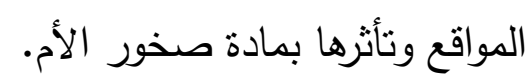
r. ان امكانية الاعتماد على تقنيات التحسس النائي والبرامجيات المرافقة لها كوسيلة حديثة في تتفيذ مشاريع

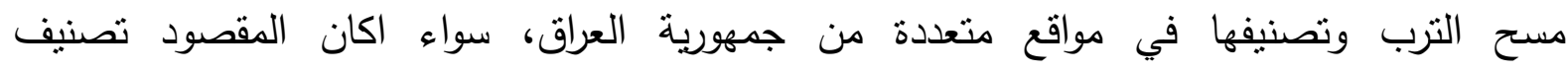

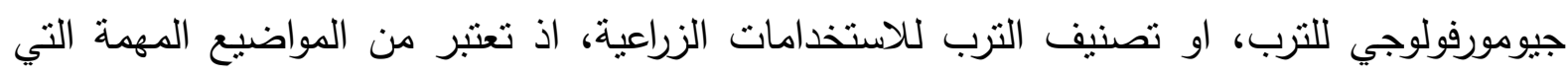

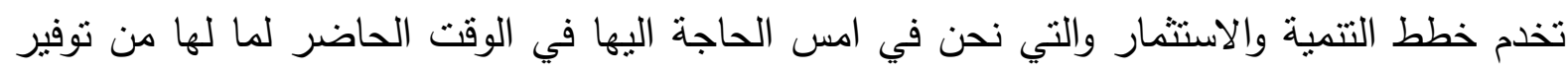
في المال والجهد والوقت. ع. ان مثل هذه الدراسات على المستوى القطري تكون بمثابة احدى الفقرات الرئيسة لبرنامج خارطة التقسيم

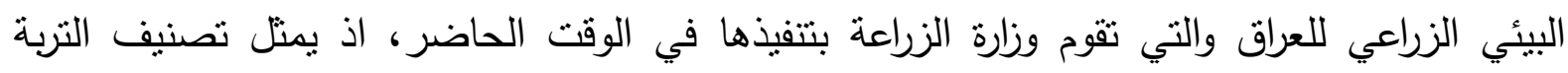

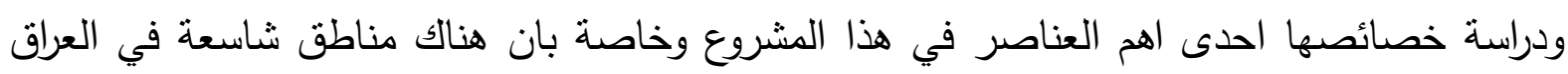
تحتاج الى مسح وتصنيف لترسبات الترب فيها على أن يؤخذ النبات النامي في عين الاعتبار .

\section{المصادر العربية}

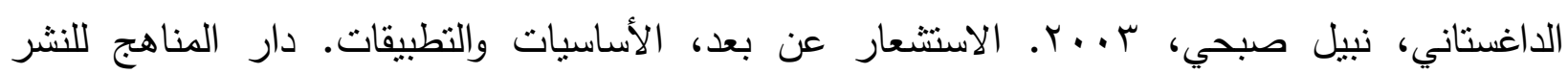

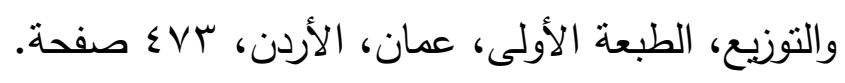

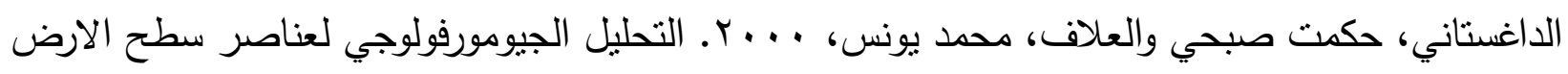

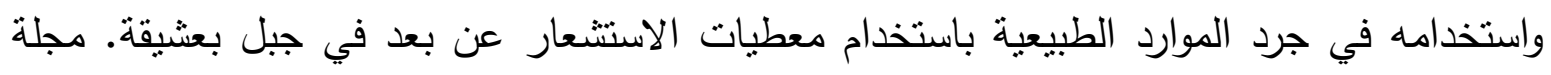

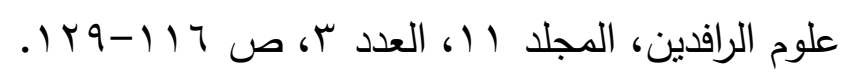

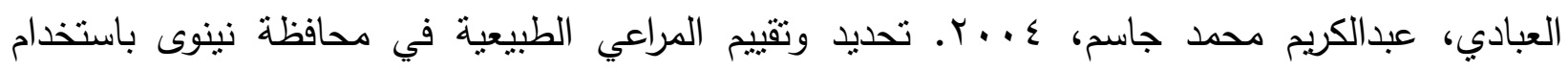

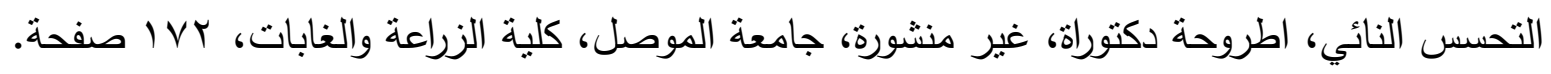

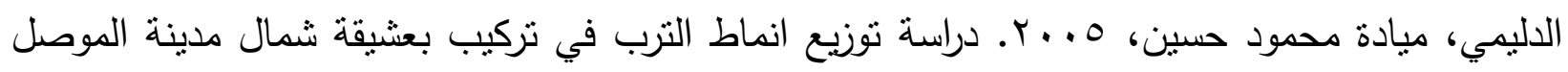

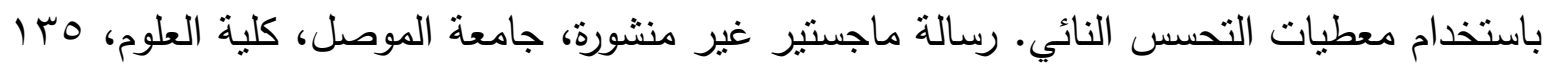



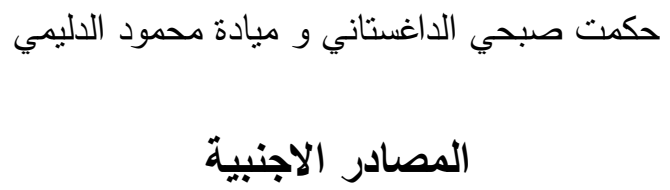

Al_Shumam, T.A.K, 2001. An Integrated Software for Remotely Sensed Data Classification, M.Sc thesis, Computer Science, University of Mosul.111p.

Biswas, T.D. and Gawande, A.C., 1964. Characteristics of Catenary Soils on GraniteGneiss Parent Rock in the Kurnool District of Andhre Pradesh .Indian Soc. Soil Sci, Vol. 14, pp.183-195.

Bruce, K.R., 1975. Geomorphic and Soil Correlation Analysis of Land Systems in Northern Zombie. Trans. Inst. Br. Geogra., pp. 67-76.

Buringh, P., 1960. Soils and Soil Condition in Iraq. Soil Survey and Classification Specialist. Ministry of Agriculture, Baghdad, Iraq,132p.

Lillesand, T.M. and Kiefer, R.W., 2000. Remote Sensing and Image Interpretation, $4^{\text {th }}$ Edition, John Wiley and Sons, 724p.

Mulder, M.A., 1978. Remote Sensing in Soil Science. Development in Soil Science, 15, Elsevier, Science, Publishers, B.V.,376p.

Nedeco, 1982. East Jazerra Irrigation Project. Final Planning Report, Vol.2,13p.

Salih, M.R. and Al-Daghastani, H.S., 1993. Thrust Mechanism and Their Relationship with Folding and Geomorphology of Bashiga Structure, North of Iraq. Iraqi Geological Journal, Vol.26, No.2, pp.62-75.

Soil Survey Staff, 1975. Soil Taxanomy; Abasic System of Soil Classification for Making and Intrpreting Soil Surveys. USA Dept. Agric. Handbook, No. 436, Washingtion.

U.S. Department of Agriculture, 1966. U.S. Aerial-Photo Interpretation in Classifying and Mapping Soil. Handbook, No. 294, Washingtion. D.C., pp.37-51. 